\title{
Assessment of extended-spectrum $\beta$-lactamases and integrons among Enterobacteriaceae in device-associated infections: multicenter study in north of Iran
}

\author{
Masoumeh Bagheri-Nesami ${ }^{1}$, Alireza Rafiei ${ }^{2}$, Gohar Eslami ${ }^{3}$, Fatemeh Ahangarkani ${ }^{4}$, Mohammad Sadegh Rezai ${ }^{*}$, \\ Attieh Nikkhah ${ }^{5}$ and Azin Hajalibeig ${ }^{1}$
}

\begin{abstract}
Background: Device-associated nosocomial infections (DA-NIs), due to MDR Enterobacteriaceae, are a major threat to patient safety in ICUs. We investigated on Extended-spectrum $\beta$-lactamases (ESBL) producing Enterobacteriaceae and incidence of integrons in these bacteria isolated from ventilator-associated pneumonia (VAP) and catheter-associated urinary tract infections (CAUTIs) in 18 governmental hospitals in the north of Iran.

Methods: In this cross-section study, the antibiotic susceptibility test was performed using the MIC method; also, phenotypically detection of ESBL-producing bacteria was carried out by the double-disk synergy (DDS) test. Presence of ESBL-related genes and integron Classes 1 and 2 was evaluated by the PCR method.

Results: Out of a total of 205 patients with DA-NIs, Enterobacteriaceae were responsible for (72.68\%) of infections. The most common DA-Nls caused by Enterobacteriaceae were VAP (77.18\%), CAUTI (19.46\%), and sepsis due to VAP (3.35\%). The most frequently Enterobacteriaceae were; Klebsiella pneumoniae 75 (24; 32\% ESBL positive), E. coli 69 (6; 8.69\% ESBL positive) and Enterobacter spp. 5 (5; 100\% ESBL positive). Distribution of ESBL-related genes was as follows: bla-SHV (94.3\%), bla-CTX (48.6\%), bla-VEB (22.9\%) and bla-GES (17.14\%). The incidence rate of integron class 1 and class 2 was (82.92\%) and (2.9\%) respectively. Eight types of ESBL-producing bacteria were observed.

Conclusions: Due to the fact that the emergence rate of ESBL Enterobacteriaceae is increasing in DA-NIs, co-incidence of different types of ESBL genes with integrons in $75-100 \%$ of strains in our study is alarming for clinicians and healthcare safety managers. Therefore, regional and local molecular level estimations of ESBLs that are agents of DA-NIs are critical for better management of empiric therapy, especially for patients in ICUs.
\end{abstract}

\section{Background}

Device-associated nosocomial infections (DA-NIs), especially ventilator-associated pneumonia (VAP) and catheterassociated urinary tract infections (CAUTIs) pose the greatest threat to patient safety in the ICUs [1-3]. VAP is the most lethal among the two, however, CAUTIs are the most common Enterobacteriaceae that have been indicated

\footnotetext{
* Correspondence: drmsrezaii49@gmail.com

${ }^{1}$ Infection Diseases Research Center with Focus on Nosocomial Infection,

Mazandaran University of Medical Sciences, Sari, Iran

Full list of author information is available at the end of the article
}

as the most common cause of extended-spectrum $\beta$ lactamases (ESBL) producing bacteria in ICUs. These bacteria have a plethora of resistance mechanisms and often use multiple mechanisms against the same antibiotic or use a single mechanism to affect multiple antibiotics. Resistance to broad-spectrum cephalosporin is spreading quickly among Enterobacteriaceae and this is mostly related to acquisition of ESBL genes. Isolates that express ESBL phenotypes and hydrolize the beta lactam antibiotics are often multiple drug resistant (MDR) $[4,5]$. The commonly genes related to the ESBL phenotype 
are sulf-hydryl variable (SHV), cefotaxime-beta lactamases (CTX), Vietnam extended-spectrum $\beta$-lactamase (VEB) and Guyana Extended-Spectrum ß-lactamases (GES) genes. Integrons as mobile DNA elements, are capable of detention and excision of antibiotic-resistant genes. Integrons achieve this by site-specific recombination. The different combinations of gene cassettes can contribute to the diverse genetic organization of integrons. There are five different classes of integrons. Class 1 integrons are the most common type that are present in Enterobacteriaceae.

Class 2 integrons are associated with the Tn7 transposon, whose transposition activity is directed at specific attachment sites on chromosomes or plasmids. Many of the antibiotic-resistant genes found in clinical isolates of Enterobacteriaceae are part of a gene cassette inserted into an integron [6]. Due to the potential of integrons to capture and collect gene cassettes, it is likely that incidence of MDR bacteria such as ESBLproducing Enterobacteriaceae, will become more prevalent in the future and integrons will continue to threaten the usefulness of antibiotics as therapeutic agents [6-8]. ESBL genes can be located on integrons, which may facilitate the spread of such genetic elements. To the best of our knowledge, this study is the first of its kind on ESBLproducing Enterobacteriaceae and incidence of integrons in these bacteria isolated from VAP and CAUTI as a major threat to patient safety in ICU wards, which was conducted in 18 governmental hospitals of Mazandaran province (The largest province in the north of Iran in terms of area and population).

\section{Methods}

\section{Study population and DA-Nls definitions}

This cross-sectional study was conducted in 18 governmental hospitals that overall contained 1200 ward beds and 100 intensive care unit beds, in Mazandaran province, located in the north of Iran, during 2014 and 2015. This study was approved by the Ethics Committee of Mazandaran University of Medical Sciences (Code No: 879 Date: July 9, 2014).

DA-NIs were defined as: Catheter-Associated Urinary Tract Infection; patient with a urinary catheter that had fever, dysuria, frequency, flank pain, suprapubic pain, nausea and vomiting. In addition, the urine culture was positive for $10^{5}$ colony forming units per $\mathrm{mL}$ or more, with no more than two microorganisms isolated or, must have had at least two symptoms such as fever, dysuria, frequency, flank pain, suprapubic pain, nausea and vomiting plus pyuria.

Ventilator-Associated Pneumonia; Ventilator-associated pneumonia was indicated in a mechanically ventilated patient with a chest radiograph that showed new or progressive infiltrates, cavitation, consolidation, or pleural effusion $48 \mathrm{~h}$ after hospitalization. The patient must have had at least one of the following criteria: new onset of purulent sputum or change in character of sputum; organism cultured from blood or from a specimen obtained by tracheal aspirate, bronchoalveolar lavage or bronchial brushing, or biopsy.

Sepsis due to VAP; in patients ventilated more than $72 \mathrm{~h}$, and bacteria separated from positive blood culture and tracheal tube aspirate positive culture were similar; while the patients had the symptoms of systemic inflammatory response syndrome (SIRS).

For all the patients whom were subject to ventilator and urinary catheter, certain prevention strategies were used against VAP and CAUTIs.

The prevention strategies for CAUTIs: insert catheters just for appropriate indications; leave catheters only as long as needed; only trained nurses insert and maintain catheters; hand hygiene; Insert catheters using aseptic technique and sterile equipment; aseptic insertion, maintain a closed drainage system; maintain unobstructed urine flow.

The prevention strategies for VAP are: elevation of the head of the bed; oral hygiene care; Prophylaxis interventions for peptic ulcer disease and deep vein thrombosis.

\section{Sampling and microbiological methods}

For VAP, a deep tracheal aspirate from the endotracheal tube was obtained, and for CAUTI, urine was aseptically aspirated from the sampling port of the urinary catheter for performing gram stain and culture on selective media. Sampling was done by the head nurses and the samples were immediately transported in a transport medium to the microbiology laboratory. All the samples were routinely cultured on MacConkey and blood agar plates. Blood samples were cultured in Blood culture bottles. Isolates were identified at the species level using standard biochemical tests and microbiological methods $[9,10]$.

\section{Antibiotic susceptibility test}

Susceptibility of the clinical isolates to routinely used antibiotics was determined by the standard broth dilution (micro dilution) technique. MIC was determined according to the recommendations of the standard protocol of CLSI 2010. The antibiotics were purchased from Sigma chemical company. Antibiotics used in this study were Amikacin, Ciprofloxacin, Imipenem, Gentamicin, Ceftazidime, Tobramycin, Piperacillin-Tazobactam, Cefepime, Colistin and Co-trimoxazole.

\section{Phenotype detection of extended-spectrum beta-lactamase (ESBL) producing enterobacteriaceae} ESBL-producing Enterobacteriaceae was detected using the double-disk synergy (DDS) test [11, 12]. ESBL's presence was assayed using the following antibiotic 
disks (MAST, UK): cefotaxime (30 $\mu \mathrm{g})$, cefotaxime/clavulanic acid $(30 / 10 \mu \mathrm{g})$, ceftazidime $(30 \mu \mathrm{g})$, and ceftazidime/clavulanic acid (30/10 $\mu \mathrm{g})$. Escherichia coli ATCC 25922 strain served as positive controls.

\section{DNA extraction and detection of ESBL-related genes}

Enterobacteriaceae that were phenotypically confirmed as ESBL, were evaluated for ESBL-related genes. DNA of ESBL-positive Enterobacteriaceae was extracted using a commercial gene extraction kit (DNA Zist, Iran) according to the company's instructions. ESBL-positive strains were screened by the PCR method for genes bla CTX, bla VEB, bla GES, bla SHV and also integrons class 1 and class 2 . The set of primers and PCR amplification conditions are available in Additional file 1. After performing the PCR reaction, electrophoresis of PCR products was carried out in $2 \%$ agarose gel at 70 voltage for $50 \mathrm{~min}$. Then, results were evaluated under UV light on the UV Trans illuminator. In all the experiments, the following reference strains were used as positive controls: K. pneumoniae 7881 (CTXM), K. pneumoniae 7881 strain (containing SHV), P. aeruginosa ATCC 27853 (VEB-1), and K. pneumoniae (GES). E. coli $96 \mathrm{~K} 062$ was used as a positive control for classes 1 and 2 integrons. A non-ESBL-producing strain (E. coli ATCC 25922) was used as negative control.

\section{Statistical analysis}

Data were analyzed using SPSS software version 16. Descriptive statistics, Chi- square and Fisher's exact tests were used for statistical analysis.

\section{Results}

Out of total of 205 hospitalized patients with DA-NIs in ICU wards of the mentioned hospitals during 2014-2015, Enterobacteriaceae were responsible of 149 (72.68\%) of DA-NIs. The most frequently found Enterobacteriaceae were; Klebsiella pneumoniae 75 (24; 32\% ESBL positive \& 51; 68\% ESBL negative), E. coli 69 (6; 8.69\% ESBL positive \& 63; 91.30\% ESBL negative) and Enterobacter spp. 5 (5; 100\% ESBL positive). The most common DA-NIs caused by Enterobacteriaceae were VAP (77.18\%), CAUTI (19.46\%) and sepsis due to VAP (3.35\%).

The demographic feature of patients with DA-NIs caused by ESBL Enterobacteriaceae was as follows; 27 VAP patients $(15 ; 55.5 \%$ male and $12 ; 44.4 \%$ female) with average age of $66.5 \pm 20.17$ years and average duration of hospitalization in the ICU of $28.37 \pm 20.03$ days; three CAUTI patients ( $1 ; 33.3 \%$ male and $2 ; 66.6 \%$ female) with average age of $45.66 \pm 21.93$ years and average duration of hospitalization in the ICU of $19.33 \pm 15.63$ days; five patients of sepsis due to VAP $(2 ; 40 \%$ male and $3 ; 60 \%$ female) with average age of $60.25 \pm 14.79$ years and average duration of hospitalization in the ICU of $10.75 \pm 1.89$ days.

In total, the distribution of ESBL-related genes was 33 (94.3\%) bla-SHV, 17 (48.6\%) bla-CTX, 8 (22.9\%) blaVEB and $6(17.14 \%)$ bla-GES. Figure 1 that is the illustration of agarose gel, shows the strains containing VEB, SHV, int1 (integron class1), int2 (integron class 2), GES and CTX genes. The Antibiotic susceptibility pattern of Enterobacteriaceae containing ESBL-related genes is shown in detail in Table 1. The rate of antibiotic resistance among strains containing SHV gene was 27.3-78.8\% whereas the rate of sensitivity was $6.1-48.5 \%$. On the other hand, Gentamicin and Imipenem had the highest resistance and sensitivity rates respectively. The rate of antibiotic resistance among strains containing CTX gene was $41.2-88.2 \%$, whereas the rate of sensitivity was $5.9-35 \%$. In addition, Gentamicin and Ciprofloxacin had the highest resistance and sensitivity rates respectively. The rate of antibiotic resistance among strains containing VEB gene was $12.5-87 \%$, whereas the rate of sensitivity was 12.5-75\% and Ceftazidime and Ciprofloxacin had the highest resistance and sensitivity rates respectively. The rate of antibiotic resistance among strains containing

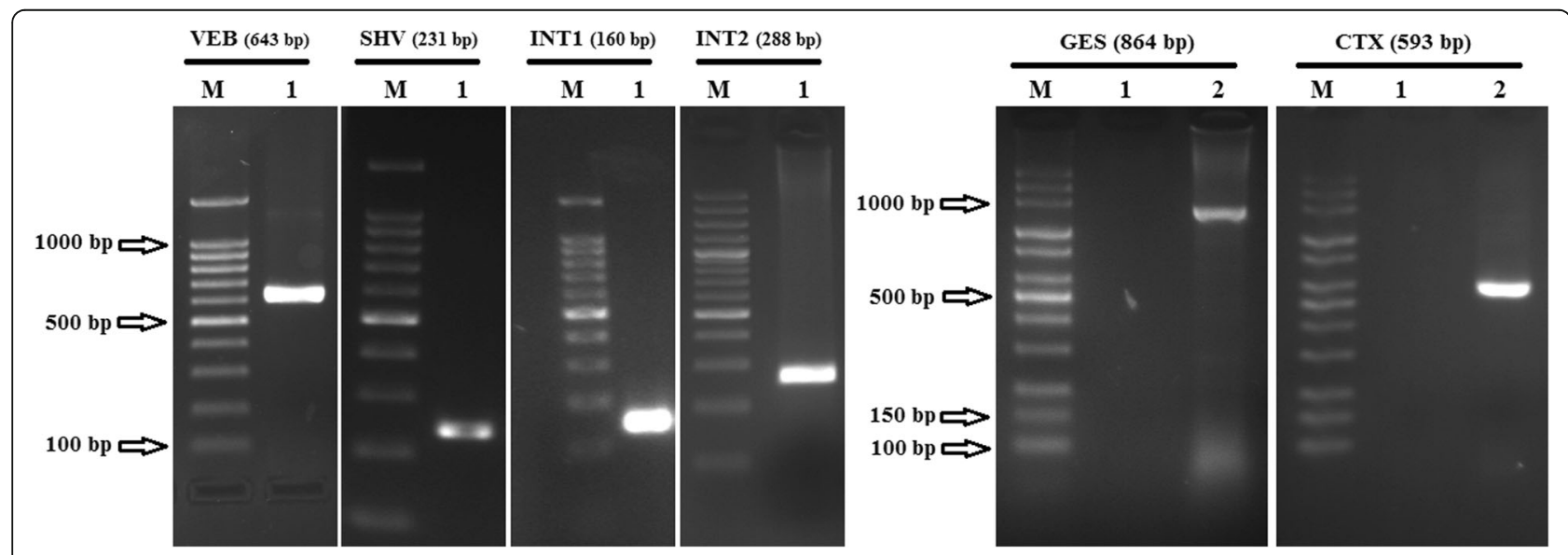

Fig. 1 Agarose gel showing the strains containing, VEB, SHV, int1 (integron class1), int2 (integron class 2), GES and CTX genes 
Table 1 Antibiotic susceptibility pattern of Enterobacteriaceae containing ESBL related genes

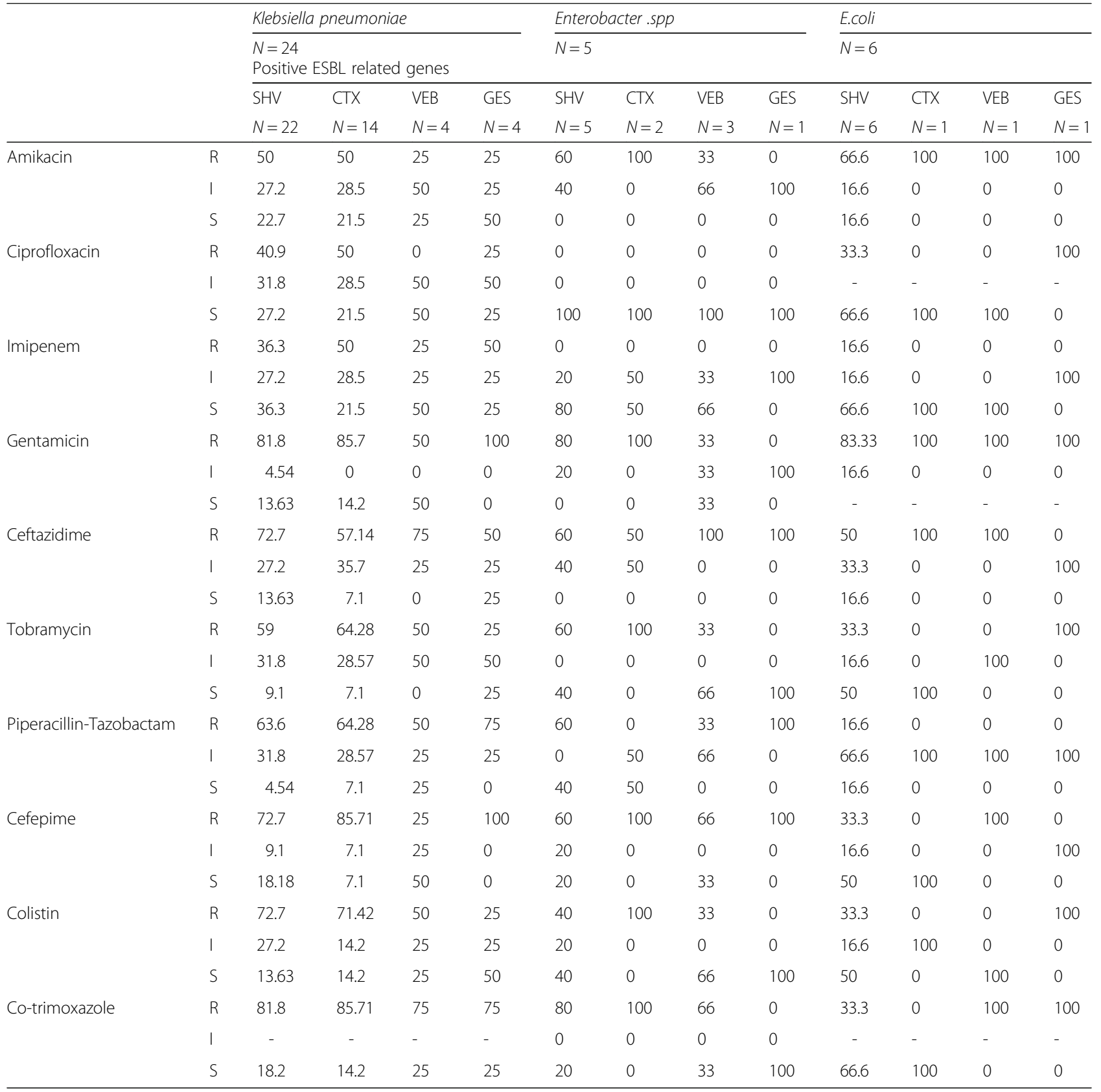

$R$ resistant, $/$ intermediate, $S$ sensitive

GES gene was $16.7-83.3 \%$ whereas the rate of sensitivity was $16.7-66.7 \%$. On the other hand, Cefepime and Imipenem had the highest resistance and sensitivity rates respectively. The incidence of integrons class 1 and class 2 was $29(82.92 \%)$ and $1(2.9 \%)$ respectively. Antibiotic susceptibility pattern of integron-positive Enterobacteriaceae is shown in Table 2.

The rate of antibiotic resistance among integron class 1 positive strain was $35-85 \%$. The only integron class 2 positive strain was Klebsiella pneumoniae and this isolate was resistant to all the antibiotics. Eight types of ESBL genes were seen among the isolates. Coincidence of each type of ESBL-producing bacteria and integron class 1 is shown in Table 3. Nine strains contained three ESBL genes (2 strains had GES, VEB, and SHV, 3 strains had GES, CTX, and SHV and 4 strains had VEB, CTX, and SHV). Fourteen strains contained 2 ESBL genes (10 strains had CTX and SHV, one strain had GES and SHV and three strains had VEB and SHV). Twelve strains had only one ESBL gene (11 strains contained 
Table 2 Antibiotic susceptibility pattern of integron positive Enterobacteriaceae

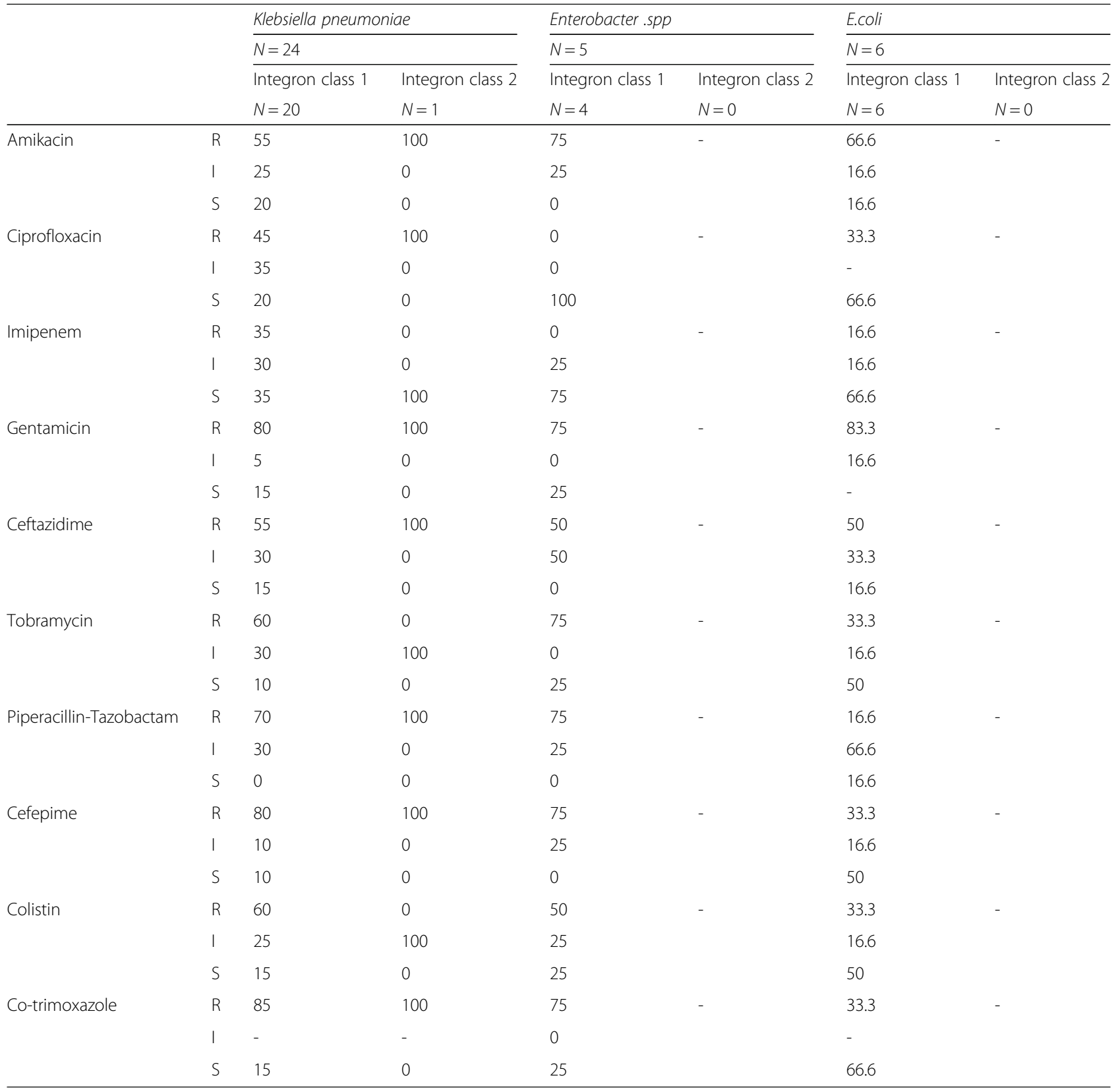

$R$ resistant, $I$ intermediate, $S$ sensitive

SHV and one strain had CTX). Coincidence of isolates that contained different types of ESBL genes and integron class 1 was $75-100 \%$, which was statistically significant $(P>.05)$.

\section{Discussions}

DA-NIs due to MDR bacteria are a serious threat to patient safety, being among of the most serious causes of morbidity, mortality and economic burden in developing countries such as Iran. Various studies have shown that the DA-NIs are a serious issue in Iran [13-16] But no studies had specifically addressed and evaluated the
ESBL genes and mobile genetic elements such as integrons in Enterobacteriaceae as common agents of DA-NIs patients in Iran. It was found in this study that ESBL-producing Enterobacteriaceae were causative agents of $23 \%$ of DA-NIs in the region. Rosenthal et al. surveyed DA-NIs in 55 ICUs of eight developing countries and found that VAP posed the greatest risk (41\%), followed by CVC-related bloodstream infections (30\%) and CAUTI (29\%). On the other hand, they reported Enterobacteriaceae were agents of about $27 \%$ of VAPs and $42 \%$ of CAUTIs [16]. In our study, overall $56 \%$ of Enterobacteriaceae were resistant to 
Table 3 Coincidence of ESBL genes types and integron class 1 among Enterobacteriaceae isolated

\begin{tabular}{llll}
\hline ESBL types & Number & $\begin{array}{l}\text { Coincidence with } \\
\text { integron class } 1\end{array}$ & $P$ value \\
\hline GES, VEB, SHV & $2(5.71)$ & $2(100)$ & 0.00 \\
GES, CTX, SHV & $3(8.57)$ & $3(100)$ & 0.00 \\
VEB, CTX, SHV & $4(11.42)$ & $3(75)$ & 0.00 \\
CTX, SHV & $10(28.57)$ & $8(80)$ & 0.01 \\
GES, SHV & $1(2.85)$ & $1(100)$ & 0.00 \\
VEB, SHV & $3(8.57)$ & $1(100)$ & 0.00 \\
SHV & $11(31.42)$ & $11(100)$ & 0.02 \\
CTX & $1(2.85)$ & $1(100)$ & 0.00 \\
\hline
\end{tabular}

the 3rd generation of cephalosporin, and imipenem was the most effective antibiotic. Similar to the results of this study, Rosenthal et al. reported that 51\% of Enterobacteriaceae isolates were resistant to ceftriaxone [16]. Salomao et al. reported that in five ICUs in three urban hospitals of Brazil during a three-year period, VAP rate was 20.9 per 1000 ventilator days, CAUTI rate was 9.6 per 1000 catheter days, and Enterobacteriaceae were agents of $22.8 \%$ of DA-NIs. Enterobacteriaceae in their study were resistant to ceftriaxone in $96.7 \%$ of the cases and resistance to ceftazidime was seen in $79.3 \%$ of the cases [17]. GuancheGarcell in Cuba determined the incidence rate of DA-NIs to be $17.0 \%$ for VAP, $4.4 \%$ for CAUTI and $1 \%$ for CVC. They found that overall $51.7 \%$ of all DA-NIs were caused by Enterobacteriaceae. Escherichia coli was responsible for VAP and CAUTI for 15.4 and $53.8 \%$ of the cases respectively. VAP and CAUTI due to Klebsiella spp. were 23.1 and $15.4 \%$ respectively. The rate of VAP caused by Enterobacteriaceae in the mentioned studies was lower than the findings of this study.

ESBL Enterobacteriaceae pose unique challenges to infection control professionals and antibacterial-discovery scientists $[18,19]$. In this study, prevalence of ESBLrelated genes was; $94.3 \%$ for SHV, $48.6 \%$ for CTX, $22.9 \%$ for VEB and $17.14 \%$ for GES. $\beta$-Lactams not only are extensively used for treatment of common infections, but also frequently used as prophylaxis before surgery. Enterobacteriaceae are of clinical importance since they cause infections, especially in patients that use the devices in ICUs. ESBL-producing Enterobacteriaceae are often MDR, further limiting the therapeutic options. In the present study, Co-resistance with fluoroquinolones, aminoglycosides, trimethoprim, and cephalosporins were found in average in 33-80\% of ESBL bacteria. Knowledge of the local epidemiology of ESBL DA-NIs in molecular level is very important. For an immunocompromised patient, such as patients admitted in ICUs with DA-NIs caused by ESBL-producing Enterobacteriaceae, administration of an ineffective antibiotic can be lethal. The rate of ESBL varies geographically but it is increasing fast in the region; for example in our previous study on ESBL-Escherichia coli uropathogens of pediatrics in 2014, the rate of ESBL genes were SHV (44\%), CTX (28\%), VEB (8\%), and GES (0\%); but in this study, the rate of these genes were $16.6-100 \%$ among E. coli [20]. In addition, Khorshidi et al. in Kashan and Khosravi et al. in Ahvaz, reported the rates of blaSHV to be about $50 \%$, which is lower than the findings of this study $[21,22]$. The prevalence of ESBLproducing Enterobacteriaceae in Iran has been reported in different rates by phenotypic confirmatory test.

For example Behroozi et al., reported that $21 \%$ of $E$. coli and $12 \%$ of $K$. pneumonia isolates were ESBL producers; on the other hand, Feizabadi et al. reported that $72 \%$ of K. pneumonia strains isolated from Tehran hospitals were ESBL producers. Also in our previous study on bacteria isolated from patients with chronic sinusitis, the rate of ESBL-producing bacteria was 28.75-37.03\% among Enterobacteriaceae [12, 23, 24]. High prevalence of ESBL-producing Enterobacteriaceae that are agents of DA-NIS in ICUs, represent the rising problem of antibiotic resistance rate in the ICUs, which is caused by suboptimal infection control in hospitals and new mutations of resistant genes. Transmission of the genes of ESBL enzymes can occur by horizontal gene transfer. Integrons, as common genetic mobile elements, are associated with ESBL genes. In this study, the presence of class 1 integron varied from 80 to $100 \%$ among ESBLproducing species; also eight types of ESBL-producing Enterobacteriaceae were found. All these types significantly correlate with the incidence of integron class 1 $(p \geq 0.02)$. Similar to these results, association of certain beta-lactamase genes with class 1 integrons by location of bla genes within integron platforms (blaVIM, blaIMP, blaGES, blaVEB, blaCTX-M-2/-9, and blaCMY) or by sharing the same plasmid context (blaTEM and blaSHV) among Enterobacteriaceae, has been previously reported in several studies [25-28].

\section{Conclusions}

The emergence of ESBL Enterobacteriaceae among DA-NIs is increasing in the Mazandaran province. The emergence of coincidence of different types of ESBL genes with integrons in $75-100 \%$ of strains is really dangerous and alarming for clinicians and healthcare safety managers. Due to the fact that prevalence of ESBL-producing strains can vary greatly from one ward to another, and even for a given ward in different points in time, therefore estimating regional and local ESBL agents of DA-NIs in molecular level in high-risk wards, at least once a year, could be useful in clinical decision-making of empiric therapy, especially for patients in ICUs. 


\section{Additional file}

Additional file 1: Table S1. The set of primers and PCR amplification conditions. (DOCX $12 \mathrm{~kb}$ )

\section{Abbreviations}

CAUTI: Catheter-associated urinary tract infections; CTX: Cefotaxime-beta lactamases; CVC: Central venous catheter; DA-NIs: Device associated nosocomial infections; ESBL: Extended-spectrum beta-lactamases; GES: Guyana ExtendedSpectrum ß-lactamases; ICU: Intensive care unit; MIC: Minimum inhibitory concentration; NIs: Nosocomial infection; PCR: Polymerase chain reaction; SHV: Sulf-hydryl variable; VAP: Ventilator-associated pneumonia; VEB: Vietnam extended-spectrum $\beta$-lactamase

\section{Acknowledgments}

This article was a part of a specialty's thesis of Pediatric of Dr Azin Hajalibeig and was supported by the Vice-Chancellor for Research at Mazandaran University of Medical Sciences (Grant Number: 879).

\section{Funding}

Mohammad Sadegh Rezai received Research grants of Vice-Chancellor for Research at Mazandaran University of Medical Sciences with grant number 879.

\section{Availability of data and materials}

All data analysed during this study are included in this published article.

\section{Authors' contributions}

Mohammad Sadegh Rezai and Masome Bagheri Nesami designed the project, collected data and wrote the manuscript. Gohar Eslami, Azin Hajalibeig, Fatemeh Ahangarkani and Attieh Nikkhah collected data. Alireza Rafiei carried out laboratory examinations. All authors read and approved the final manuscript.

\section{Competing interests}

The authors declare that they have no competing interests.

\section{Consent for publication}

Not applicable.

\section{Ethics approval and consent to participate}

This study was approved by the Ethics Committee of Mazandaran University of Medical Sciences (Code No: 879 Date: July 9, 2014).

\section{Author details}

${ }^{1}$ Infection Diseases Research Center with Focus on Nosocomial Infection, Mazandaran University of Medical Sciences, Sari, Iran. ${ }^{2}$ Molecular and Cell Biology Research Center, Department of Immunology, Faculty of Medicine, Mazandaran University of Medical Sciences, Sari, Iran. ${ }^{3}$ Department of Clinical Pharmacy, Faculty of Pharmacy, Mazandaran University of Medical Sciences, Sari, Iran. ${ }^{4}$ Student Research Committee, Antimicrobial Resistance Research Center, Mazandaran University of Medical Sciences, Sari, Iran. ${ }^{5}$ Traditional and Complementary Medicine Research Center, Mazandaran University of Medical Sciences, Sari, Iran.

Received: 9 August 2016 Accepted: 19 October 2016

Published online: 01 December 2016

\section{References}

1. Blot $\mathrm{S}$, et al. Influence of matching for exposure time on estimates of attributable mortality caused by nosocomial bacteremia in critically ill patients. Infect Control Hosp Epidemiol. 2005;26(4):352-6.

2. Maki DG, Tambyah PA. Engineering out the risk for infection with urinary catheters. Emerg Infect Dis. 2001;7(2):342.

3. Heyland DK, et al. The attributable morbidity and mortality of ventilatorassociated pneumonia in the critically ill patient. Am J Respir Crit Care Med. 1999:159(4):1249-56.

4. Bradford PA. Extended-spectrum $\beta$-lactamases in the 21st century: characterization, epidemiology, and detection of this important resistance threat. Clin Microbiol Rev. 2001;14(4):933-51.
5. Pitout JD, Laupland KB. Extended-spectrum beta-lactamase-producing Enterobacteriaceae: an emerging public-health concern. Lancet Infect Dis. 2008;8(3):159-66.

6. White PA, Mclver CJ, Rawlinson WD. Integrons and gene cassettes in the enterobacteriaceae. Antimicrob Agents Chemother. 2001;45(9):2658-61.

7. Ploy M-C, et al. Integrons: an antibiotic resistance gene capture and expression system. Clin Chem Lab Med. 2000;38(6):483-7.

8. Machado $\mathrm{E}$, et al. Preservation of integron types among Enterobacteriaceae producing extended-spectrum $\beta$-lactamases in a Spanish hospital over a 15year period (1988 to 2003). Antimicrob Agents Chemother. 2007;51(6):2201-4.

9. Collee J, Miles R, Watt B. Tests for identification of bacteria. In: Collee JG, Fraser AG, Marmion BP, editors. Practical medical microbiology. 14th ed. Edinburgh: Churchill Livingstone; 1996. p. 131-50.

10. Koneman E, Allen S, Janda W, Schreckenberger R, Winn W. Introduction to microbiology. In: Koneman EW, Alien SD, Janda WM, Schreckenberger RC, Winn W, editors. Part II; Guidelines for collection, transport, processing, analysis, and reporting of cultures from specific specimen sources, Color atlasand textbook of diagnostic microbiology. 5th ed. Philadelphia: Lippincott; 1997. p. 121-70.

11. Jarlier $V$, et al. Extended broad-spectrum $\beta$-lactamases conferring transferable resistance to newer $\beta$-lactam agents in Enterobacteriaceae: hospital prevalence and susceptibility patterns. Rev Infect Dis. 1988;10(4):867-78.

12. Rezai M-s, et al. Multidrug resistance pattern of bacterial agents isolated from patient with chronic sinusitis. Caspian J Intern Med. 2016;7(2):114-9.

13. Afhami $\mathrm{S}$, et al. Ventilator-associated pneumonia in a teaching hospital in Tehran and use of the Iranian Nosocomial Infections Surveillance Software. East Mediterr Health J. 2013;19(10):883.

14. Askarian $\mathrm{M}$, et al. Incidence of urinary tract and bloodstream infections in Ghotbeddin Burn Center, Shiraz 2000-2001. Burns. 2003;29(5):455-9.

15. Jahani-Sherafat $S$, et al. Device-associated infection rates and bacterial resistance in six academic teaching hospitals of Iran: findings from the International Nocosomial Infection Control Consortium (INICC). J Infect Public Health. 2015;8(6):553-61

16. Rosenthal VD, et al. Device-associated nosocomial infections in 55 intensive care units of 8 developing countries. Ann Intern Med. 2006:145(8):582-91.

17. Salomao R, et al. Device-associated infection rates in intensive care units of Brazilian hospitals: findings of the International Nosocomial Infection Control Consortium. Rev Panam Salud Publica. 2008;24(3):195-202.

18. Behzadnia S, et al. Nosocomial infections in pediatric population and antibiotic resistance of the causative organisms in north of Iran. Iran Red Crescent Med J. 2014;16(2):e14562. doi:10.5812/ircmj.14562.

19. Saffar $M$, et al. Antibacterial susceptibility of uropathogens in 3 hospitals, Sari, Islamic Republic of Iran, 2002-2003. 2008.

20. Rezai MS et al. Characterization of Multidrug Resistant Extended-Spectrum Beta-Lactamase-Producing Escherichia coli among Uropathogens of Pediatrics in North of Iran. Biomed Res Int. 2015;2015. doi:10.1155/2015/309478.

21. Khorshidi A, et al. Prevalence of TEM1 \& SHV1 genes in Kelebsiella pneumoniea with ESBL. J Mil Med. 2009;11(3):149-53.

22. Khosravi AD, Hoveizavi $H$, Mehdinejad M. Prevalence of Klebsiella pneumoniae Encoding Genes for Ctx-M-1, Tem-1 and Shv-1 ExtendedSpectrum Beta Lactamases (ESBL) Enzymes in Clinical Specimens. Jundishapur J Microbiol. 2013;6(10):e8256. doi:10.5812/jjm.8256.

23. Behrooozi A, Rahbar M, Jalil V. Frequency of extended spectrum betalactamase (ESBLs) producing Escherichia coli and Klebseilla pneumonia isolated from urine in an Iranian 1000-bed tertiary care hospital. Afr J Microbiol Res. 2010;4(9):881-4.

24. Feizabadi MM, et al. Genetic characterization of ESBL producing strains of Klebsiella pneumoniae from Tehran hospitals. J Infect Dev Ctries. 2010;4(10):609-15

25. Corkill JE, Anson JJ, Hart CA. High prevalence of the plasmid-mediated quinolone resistance determinant qnrA in multidrug-resistant Enterobacteriaceae from blood cultures in Liverpool, UK. J Antimicrob Chemother. 2005;56(6):1115-7

26. Jones $L A$, et al. The aadB gene cassette is associated with blaSHV genes in Klebsiella species producing extended-spectrum $\beta$-lactamases. Antimicrob Agents Chemother. 2005:49(2):794-7.

27. Novais $\hat{A}$, et al. Dissemination and persistence of blaCTX-M-9 are linked to class 1 integrons containing CR1 associated with defective transposon derivatives from Tn402 located in early antibiotic resistance plasmids of 
IncHI2, IncP1-a, and IncFI groups. Antimicrob Agents Chemother. 2006;50(8):2741-50.

28. Sidjabat HE, et al. Identification of blaCMY-7 and associated plasmidmediated resistance genes in multidrug-resistant Escherichia coli isolated

from dogs at a veterinary teaching hospital in Australia. J Antimicrob Chemother. 2006;57(5):840-8.

Submit your next manuscript to BioMed Central and we will help you at every step:

- We accept pre-submission inquiries

- Our selector tool helps you to find the most relevant journal

- We provide round the clock customer support

- Convenient online submission

- Thorough peer review

- Inclusion in PubMed and all major indexing services

- Maximum visibility for your research

Submit your manuscript at www.biomedcentral.com/submit 